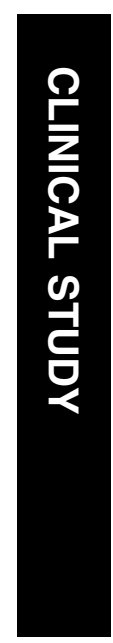

\section{Central 10-degree visual field change following trabeculectomy in advanced open-angle glaucoma}

T Fujishiro', C Mayama², M Aihara², A Tomidokoro ${ }^{2}$ and $\mathrm{M} \mathrm{Araie}^{3}$
'Saitama Red Cross Hospital, Saitama, Japan

${ }^{2}$ Department of Ophthalmology, University of Tokyo Graduate School of Medicine, Tokyo, Japan

${ }^{3}$ Kanto Central Hospital, Tokyo, Japan

Correspondence:

C Mayama, Department of Ophthalmology, University of Tokyo Graduate School of Medicine, 7-3-1, Hongo, Bunkyo-ku, Tokyo 113-8655, Japan Tel: + 81338155411 Fax: + 81338170798 E-mail: cmayama-tky@ umin.ac.jp

Received: 9 September 2010 Accepted in revised form: 15 February 2011 Published online: 15 April 2011

\begin{abstract}
Purpose To prospectively examine changes in the central visual field (VF) in patients with advanced open-angle glaucoma (OAG) with advanced late stage after trabeculectomy for 12 months.

Design Prospective interventional case series. Methods In all, 27 eyes of $27 \mathrm{OAG}$ patients at a single center with a best-corrected visual acuity (BCVA) of $\geq 40 / 200$ and a mean total deviation of test locations of the 10-2 program of the Humphrey VF analyzer of $\leq-20 \mathrm{~dB}$ preoperatively were enrolled. Intraocular pressure (IOP), VF parameters of the 10-2 program, and BCVA were examined for 12 months after trabeculectomy with mitomycin C. Slopes of VF parameters and their correlation with presumed risk factors were studied. Results IOP decreased from $19.7 \pm 5.8$ to $9.7 \pm 2.6 \mathrm{~mm} \mathrm{Hg}(P<0.001)$ over postoperative 1 year. The slopes of all VF parameters did not significantly differ from zero $(P>0.33)$, and none of the presumed factors significantly correlated with the slopes of those parameters $(P>0.14)$. There were two eyes $(7 \%)$ and one eye $(4 \%)$ with $\geq 2$ lines of deterioration in BCVA (decimal fraction) at 1 and 12 months, respectively, after surgery with no apparent causes.

Conclusions Trabeculectomy resulted in little change in the central 10-degree VF, but significant decrease in BCVA without apparent causes might occur approximately $5 \%$ of the cases.

Eye (2011) 25, 866-871; doi:10.1038/eye.2011.74; published online 15 April 2011
\end{abstract}

Keywords: glaucoma; trabeculectomy; visual field; wipe-out
Introduction

Preservation of the central visual field (VF) and visual acuity (VA) is the ultimate goal of glaucoma treatment, especially in the advanced stages of the disease. In patients with advanced glaucomatous VF damage undergoing filtration surgery, there is a risk of unpredictable and sudden postoperative deterioration of the central VF and VA, ${ }^{1-4}$ but the actual frequency of this phenomenon is controversial. ${ }^{1,2,4-8}$ Postoperative hypotony, macular splitting, and a spike in intraocular pressure (IOP) just after surgery are reportedly risk factors for the postoperative loss of central VF including the fixation point. . $^{2,3}$

Several reports have addressed this issue in advanced glaucoma, however, the study design was retrospective and VF was examined using Goldmann perimetry, which is not suitable for detailed examination of the central VF. ${ }^{1-7}$ Only one prospective report studied 21 eyes with advanced stage glaucoma using the 30-2 program of the Humphrey VF analyzer (HFA; Carl Zeiss Meditec, Jena, Germany), with a postoperative follow-up of 3 months. ${ }^{8}$ Analysis of the central VF with the 10-2 program and a longer follow-up period should provide more detailed information about the effects of surgical IOP reduction on the remaining central VF in eyes with the advanced stage of glaucoma, but there have been no such prospective studies.

The aim of this study is to prospectively examine the remaining central VF in patients with advanced open-angle glaucoma (OAG) after trabeculectomy with MMC using the HFA 10-2 program over 12 months after trabeculectomy. The influence of presumed 
relating factors on the remaining 10-degree central VF was also analyzed.

\section{Subjects and methods}

\section{Subjects}

Subjects for whom trabeculectomy with MMC was planned between May 2001 and March 2004 at the Department of Ophthalmology, University of Tokyo Graduate School of Medicine that met the following inclusion criteria were enrolled in this study after written informed consent for participation in the study was obtained. The protocol was approved by the institution's ethics committee. The inclusion criteria were (1) OAG eyes including both those with elevated and normal IOP; (2) absence of other coexisting ocular or systemic disorders, including cataract, that may affect the results of the VF or VA testing; (3) three or more reproducible VF test results taken every 6 months with the HFA 10-2 SITA standard or full threshold program before the trabeculectomy, and mean total deviation (TD) of the 10-2 program $\left(\mathrm{MTD}_{\text {all }}\right.$ ) of $\leq-20 \mathrm{~dB}$ (Figure 1) and mean deviation (MD) of the 30-2 program of $\leq-18 \mathrm{~dB}$ with a reliable preoperative test performed within 1 month before surgery; (4) preoperative best-corrected VA (BCVA) $\geq 40 / 200$ and foveal threshold (FT) $\geq 10 \mathrm{~dB}$.

Eyes with a history of previous filtering, cataract, or laser surgery were included, but eyes with any other kinds of ocular surgeries were excluded. Considering the significant portion of the current subjects had VA worse than $20 / 40$, VF test results with fixation loss, false negative, and false positive $\leq 33 \%$ were accepted.

In all, 48 consecutive eyes of 43 glaucoma patients with advanced central VF damage underwent trabeculectomy during the enrollment period. Two eyes with secondary glaucoma, 4 eyes with primary angle closure glaucoma, and 10 eyes with unreliable VF test results were excluded. Five patients met the inclusion criteria bilaterally, and an only eye with worse MTD $_{\text {all }}$ in the preoperative VF test, was included. Finally, 27 eyes of 27 OAG patients (18 men and 9 women) were enrolled in the study.

\section{Trabeculectomy and clinical follow-up}

Baseline ophthalmic examinations were performed within 1 month before the trabeculectomy. Measurement of equivalent spherical diopter power (refraction) and BCVA, slit-lamp examination, Goldmann applanation tonometry, VF tests with the 30-2 and 10-2 SITA standard or full threshold programs, and dilated funduscopy were a

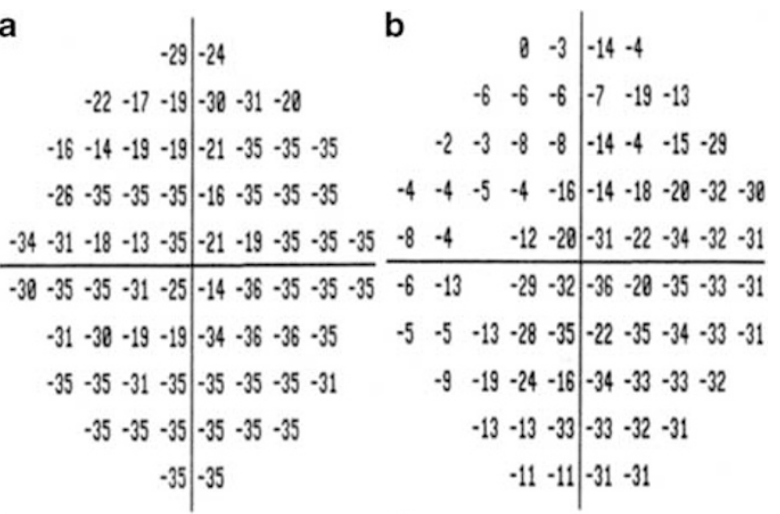

C
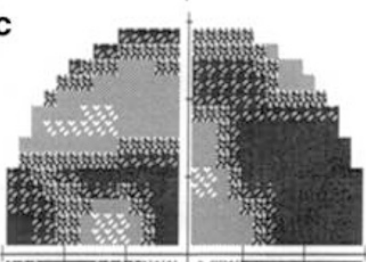

d
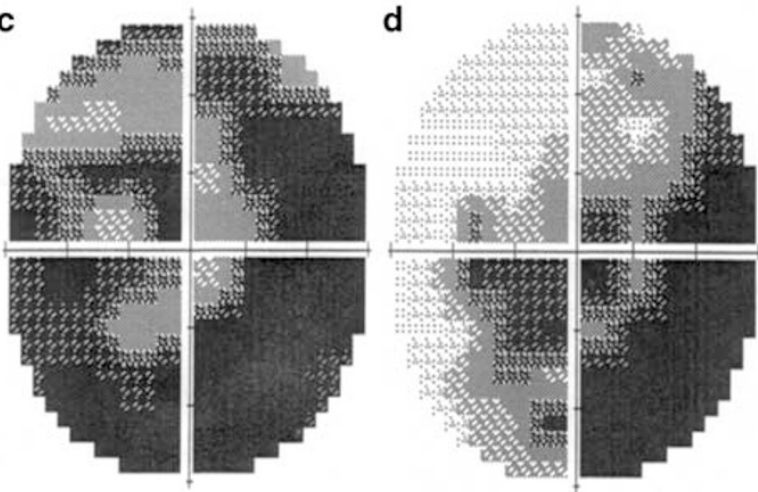

Figure 1 An example of typical preoperative VF of a subject (left eye). (a) Threshold values of 10-2 program. (b) Threshold values of 30-2 program. (c) Grayscale of 10-2 program. (d) Grayscale of 30-2 program.

performed. The reliability of the VF test was checked according to the above mentioned criteria, and VFs with unreliable test results or those with incompatible results with the previous ones were re-tested. The VF test program (SITA standard or full threshold) was uniform in every subject during the study period.

The surgery was performed by one surgeon (MA) and surgical technique was same in every subject.

Trabeculectomy was performed with a limbus-based conjunctival flap. After a triangle-shaped $(3.5 \times 3.5 \mathrm{~mm})$ scleral flap was prepared, surgical absorbent sponge pieces soaked in $0.5 \mathrm{ml}$ mitomycin-C $(0.04 \%)$ solution prepared just before use were placed between the episclera and conjunctival flap for $3 \mathrm{~min}$. After removal of the sponge pieces, the site was rinsed with $200 \mathrm{ml}$ physiologic saline. Following resection of a $1 \times 2.5 \mathrm{~mm}$ sclerocorneal block and peripheral iridectomy, the flap was closed with 10-0 nylon sutures, which were adjusted so that a little leakage around the margin of the flap was observed without decreasing the depth of the anterior chamber. Topical antibiotics were routinely administrated for up to 4 weeks and topical steroids for 
up to 12 weeks after surgery. If necessary, laser suture lysis or needle bleb revision was performed.

The subjects were examined every day during the first postoperative week, at 2 weeks, and 1 month postoperatively, and thereafter every month during the study period. IOP measurements and slit-lamp examinations were performed at each visit and associated complications such as shallow anterior chamber, choroidal detachment, or wound leakage were checked. Refraction and BCVA were measured at 1, 3, 6, and 12 months after surgery. If IOP was $<5 \mathrm{~mm} \mathrm{Hg}$ for $>1$ week during the first postoperative month, the condition was defined as short-term postoperative hypotony. When IOP was $<5 \mathrm{~mm} \mathrm{Hg}$ for $>2$ consecutive months, excluding the first postoperative month, the condition was defined as long-term postoperative hypotony. IOP reduction ${ }_{2 \mathrm{~W}}$, IOP reduction ${ }_{1 \mathrm{M}}$, IOP reduction $_{3 \mathrm{M}}$, IOP reduction ${ }_{6 \mathrm{M}}$, IOP reduction ${ }_{9 \mathrm{M}}$, and IOP reduction $_{12 \mathrm{M}}$ were defined as the difference between the mean preoperative IOP recorded within 1 month before surgery and the mean IOP recorded during the corresponding period; that is, at 1 and 2 weeks, 2 weeks and 1 month, 2 and 3 months, 4, 5 and 6 months, 7, 8 and 9 months, and 10, 11 and 12 months, respectively.

After the surgery, VF tests with the 10-2 program were scheduled at 3,6 , and 12 months by the same test strategy, SITA standard or full threshold, as the preoperative test. The eyes were re-tested when reliability of the VF test results did not meet the aforementioned criteria. Data obtained from left eyes were converted to the mirror images.

FT measured with the 10-2 program, mean TD in the whole central 10-degree VF $\left(\mathrm{MTD}_{\text {all }}\right)$, in the superior hemifield $\left(\mathrm{MTD}_{\text {sup }}\right)$ and in the inferior hemifield $\left(\mathrm{MTD}_{\mathrm{inf}}\right)$, mean TD in the four test locations closest to the fixation $\left(\mathrm{MTD}_{4}\right)$, and TD in each of the four test locations closest to the fixation (by convention designated as $\mathrm{TD}_{29}, \mathrm{TD}_{30}, \mathrm{TD}_{39}$, and $\mathrm{TD}_{40}$; Figure 2) were used for the analyses.

\section{Method of data analysis}

Refraction, BCVA, IOP, FT, MTD ${ }_{\text {all }}, \mathrm{MTD}_{\text {sup }}, \mathrm{MTD}_{\text {inf }}$, $\mathrm{MTD}_{4}, \mathrm{TD}_{29}, \mathrm{TD}_{30}, \mathrm{TD}_{39}$, and $\mathrm{TD}_{40}$ at each follow-up examination were compared with the preoperative corresponding data (ANOVA and paired $t$-test).

Similarly, the slope of FT, MTD all, $\mathrm{MTD}_{\text {sup }}, \mathrm{MTD}_{\text {inf }}$, $\mathrm{MTD}_{4}, \mathrm{TD}_{29}, \mathrm{TD}_{30}, \mathrm{TD}_{39}$, or $\mathrm{TD}_{40}$, was calculated for each eye using the preoperative baseline and results at 3,6, and 12 months after surgery by linear regression analysis. The statistical significance of slopes of those parameters was examined individually.

Spearman's correlation coefficients of the slope of FT, MTD ${ }_{\text {all }}, \mathrm{MTD}_{\text {sup }}, \mathrm{MTD}_{\text {inf }}, \mathrm{MTD}_{4}, \mathrm{TD}_{29}, \mathrm{TD}_{30}$,

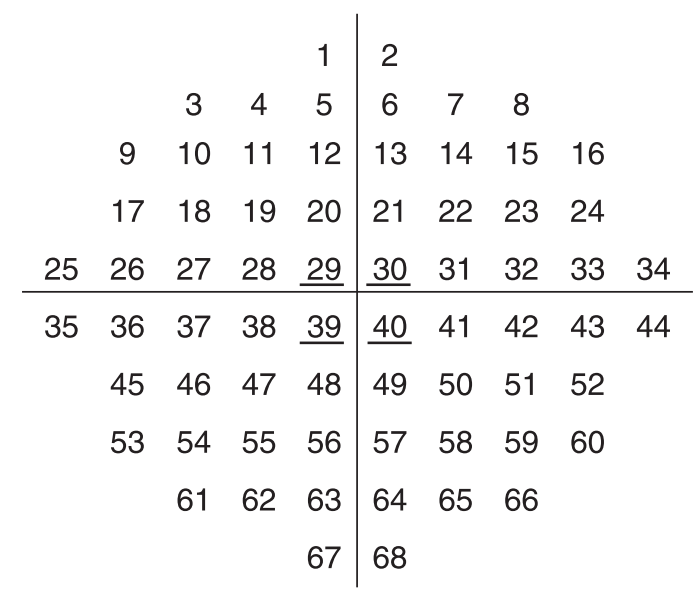

Figure 2 Numbers of VF test locations of the 10-2 program (right eye). Underlined numbers indicate test locations included in the point-wise analysis.

$\mathrm{TD}_{31}, \mathrm{TD}_{39}$, or $\mathrm{TD}_{40}, \mathrm{TD}_{41}$, or $\mathrm{TD}_{42}$ with age (year), refraction (diopter), preoperative value of each parameter, preoperative IOP, IOP reduction ${ }_{2 \mathrm{~W}}$, IOP reduction $_{1 \mathrm{M}}$, IOP reduction $3 \mathrm{M}$, IOP reduction $6 \mathrm{M}$, IOP reduction ${ }_{9 \mathrm{M}}$, IOP reduction $_{12 \mathrm{M}}$, preoperative $\mathrm{MTD}_{\text {all, }}$ and preoperative MD of the 30-2 program were calculated.

Correlation of the slope of VF parameters with the above factors were calculated for the eyes examined by the 10-2 SITA standard program and those examined by the 10-2 full threshold program combined. Statistical analyses were performed using SPSS (version 11.5, SPSS Inc., Chicago, IL, USA) and a $P$-value $<0.05$ was considered to be statistically significant.

\section{Results}

All 27 eyes of the 27 OAG patients fulfilling the inclusion criteria completed the planned follow-up of 12 months. Within 1 month after surgery, there were nine eyes with a shallow anterior chamber, six with short-term hypotony, seven with choroidal detachment, and one with wound leakage. At 1 month after surgery, all eyes recovered from the above early postoperative complications, except one eye suffered from long-term hypotony and recovered at 3 months after surgery. No eyes showed hypotonic change in the fundus and no significant cataract progression was observed in the phakic eyes according to Lens opacities classification system ${ }^{9}$ during the 12 -month study period.

The results of the preoperative examinations are summarized in Table 1. The time course of IOP is shown in Figure 3a, and IOP at 12 months after surgery was 
Table 1 Baseline characteristics of the 27 eyes of the 27 subjects

\begin{tabular}{lc}
\hline Age (years) & $54 \pm 16$ (28 to 80$)$ \\
Gender (male/female) & $18 / 9$ \\
Lens status (phakic/pseudophakic) & $6 / 21$ \\
Number of topical antiglaucoma & $3.3 \pm 0.7$ \\
agent used before trabeculectomy & \\
Equivalent refraction (diopter) & $-5.0 \pm 4.8$ \\
BCVA (log MAR) & $0.24 \pm 0.26(-0.08$ to 1.0$)$ \\
BCVA (decimal fraction) & $0.7(0.2 \text { to } 1.2)^{*}$ \\
IOP (mm Hg) & $19.7 \pm 5.8(12$ to 42$)$ \\
& \\
FT of 10-2 program $(d B)$ & $26.2 \pm 5.5(18$ to 36$)$ \\
SITA standard $(n=13)$ & $26.3 \pm 6.1(14$ to 48$)$ \\
Full threshold $(n=14)$ & \\
& \\
MTD all of 10-2 program $(d B)$ & $-25.9 \pm 3.1(-31.5$ to -20.9$)$ \\
SITA standard $(n=13)$ & $-26.7 \pm 3.6(-31.7$ to -22.5$)$ \\
Full threshold $(n=14)$ & \\
& \\
MD of 30-2 program $(d B)$ & $-23.5 \pm 4.0(-30.8$ to -19.1$)$ \\
SITA standard $(n=13)$ & $-25.5 \pm 3.4(-29.7$ to -18.7$)$ \\
Full threshold $(n=14)$ &
\end{tabular}

Abbreviations: BCVA, best-corrected visual acuity; FT, foveal threshold; IOP, intraocular pressure; MD, mean deviation; MTD $_{\text {all, }}$ mean total deviation of all test locations of the 10-2 program.

Values are mean \pm SD (range), except ${ }^{*}$ means median (range).

$9.7 \pm 2.6 \mathrm{~mm} \mathrm{Hg}$, which is significantly lower than the preoperative value $(P<0.001$, paired $t$-test).

Mean BCVA $(\log$ MAR) and MTD all showed no significant change during the 12-month follow-up (Figures $3 \mathrm{~b}$ and $\mathrm{c}, P>0.05$, ANOVA). No eyes showed $>3$ lines of deterioration in BCVA (decimal fraction) compared with the preoperative BCVA during the follow-up period. Three lines of deterioration in BCVA (decimal fraction) was found in only one (4\%) eye at 12 months and two lines of deterioration was found only at 1 month after surgery in two (7\%) eyes with no apparent cause.

Postoperative refraction, FT, $\mathrm{MTD}_{\text {all }}, \mathrm{MTD}_{\text {sup }}$, $\mathrm{MTD}_{\text {inf }}, \mathrm{MTD}_{4}, \mathrm{TD}_{29}, \mathrm{TD}_{30}, \mathrm{TD}_{39}$, and $\mathrm{TD}_{40}$ at each follow-up examination showed no significant difference from the preoperative baselines $(P>0.27$, paired $t$-test).

Slopes of the VF parameters in eyes examined with the SITA standard and the full threshold program were analyzed separately. None of the slopes of FT, MTD all, $\mathrm{MTD}_{\text {sup }}, \mathrm{MTD}_{\text {inf }}, \mathrm{MTD}_{4}, \mathrm{TD}_{29}, \mathrm{TD}_{30}, \mathrm{TD}_{39}$, and $\mathrm{TD}_{40}$ showed significant difference from 0 over the study period $(P>0.05$; Table 2$)$. In the individual analyses, none of the eyes showed the slope significantly different from 0 after correlation of multiplicity $(P<0.05, n=13-14)$ in any of the VF parameters.

The correlation of the slopes of VF parameters with preoperative and postoperative factors was studied
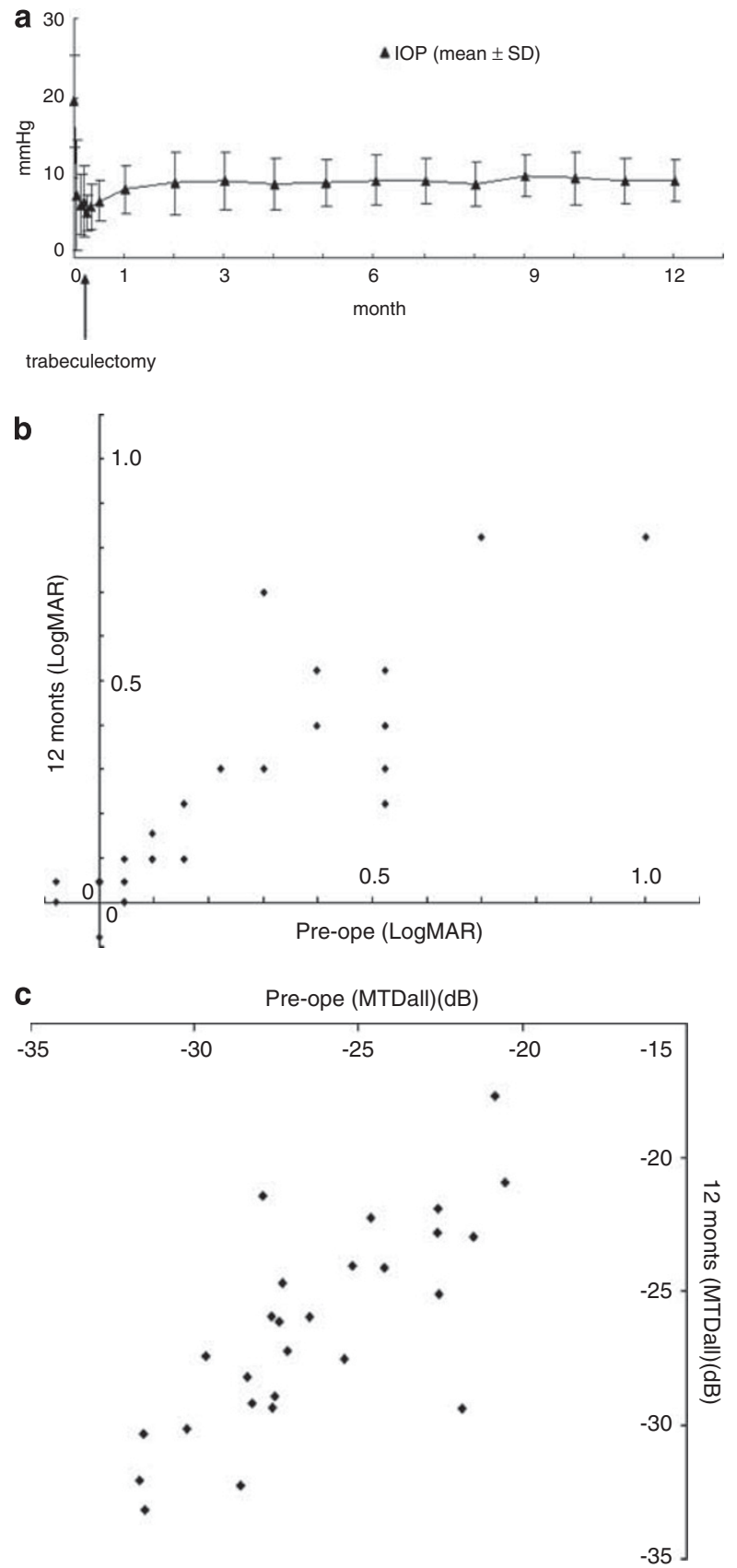

Figure 3 (a) Time course of IOP over the study period. (b) A scatter plot of preoperative BCVA vs that at 12 months after surgery. (c) A scatter plot of preoperative mean TD of all test locations of the 10-2 program (MTD all $)$ vs that at 12 months after surgery.

using the pooled results from both SITA standard and full threshold program-tested group $(n=27)$. Slopes of FT, MTD all $_{1}, \mathrm{MTD}_{\text {sup }}, \mathrm{MTD}_{\text {inf }}, \mathrm{MTD}_{4}, \mathrm{TD}_{29}, \mathrm{TD}_{30}, \mathrm{TD}_{39}$, and $\mathrm{TD}_{40}$ showed no significant correlation with age, 
Table 2 Slopes of visual field parameters over the study period

\begin{tabular}{|c|c|c|c|}
\hline Parameter & Visual field test & Slope (dB/year) Mean (95\% CI) & $\begin{array}{c}\text { Number of eyes having significant slope } \\
\text { (positive/negative), P-value* }\end{array}$ \\
\hline \multirow[t]{2}{*}{ FT } & SITA $(n=13)$ & $1.58(-3.77: 6.95)$ & 0 \\
\hline & Full $(n=14)$ & $-2.48(-3.40: 8.36)$ & 2 (negative), $P=0.02-0.03$ \\
\hline \multirow[t]{2}{*}{$\mathrm{MTD}_{\text {sup }}$} & SITA $(n=13)$ & $1.50(-5.55: 8.55)$ & 0 \\
\hline & Full $(n=14)$ & $-1.16(-2.90: 0.58)$ & 0 \\
\hline \multirow[t]{2}{*}{$\mathrm{MTD}_{\mathrm{inf}}$} & SITA $(n=13)$ & $1.14(-6.86: 9.63)$ & 0 \\
\hline & Full $(n=14)$ & $0.42(-1.03: 1.87)$ & 1 (negative), $P=0.04$ \\
\hline \multirow[t]{2}{*}{$\mathrm{MTD}_{\text {all }}$} & SITA $(n=13)$ & $1.32(-3.16: 5.80)$ & 0 \\
\hline & Full $(n=14)$ & $-0.37(-1.66: 0.91)$ & 1 (negative), $P=0.01$ \\
\hline \multirow{2}{*}{$\mathrm{MTD}_{4}$} & SITA $(n=13)$ & $0.94(-5.93: 7.79)$ & 0 \\
\hline & Full $(n=14)$ & $-1.25(-11.08: 8.58)$ & 1 (negative), $P=0.05$ \\
\hline \multirow[t]{2}{*}{$\mathrm{TD}_{29}$} & SITA $(n=13)$ & $2.29(-1.09: 5.69)$ & 1 (positive), $P=0.02$ \\
\hline & Full $(n=14)$ & $-2.57(-4.63:-0.50)$ & 0 \\
\hline \multirow[t]{2}{*}{$\mathrm{TD}_{30}$} & SITA $(n=13)$ & $-0.07(-2.78: 0.95)$ & 1 (positive), $P=0.02$ \\
\hline & Full $(n=14)$ & $-3.71(-28.03: 20.60)$ & 1 (negative), $P=0.03$ \\
\hline \multirow[t]{2}{*}{$\mathrm{TD}_{39}$} & SITA $(n=13)$ & $2.57(-6.35: 11.48)$ & 1 (positive), $P=0.02$ \\
\hline & Full $(n=14)$ & $-0.67(-9.04: 7.69)$ & 1 (negative), $P=0.05$ \\
\hline \multirow[t]{2}{*}{$\mathrm{TD}_{40}$} & SITA $(n=13)$ & $1.60(-9.91: 13.10)$ & 1 (positive), $P=0.03$ \\
\hline & Full $(n=14)$ & $-0.76(-13.12: 11.62)$ & 1 (negative), $P=0.04$ \\
\hline
\end{tabular}

Abbreviations: CI, confidential interval; FT, foveal threshold; Full, 10-2 full threshold program; MTD all, mean total deviation of all test points of the 10-2 program; SITA, 10-2 SITA standard program; TD, total deviation.

${ }^{*} P$-values without correction for multiplicity.

refraction, and any of the preoperative and postoperative factors.

\section{Discussion}

The prevalence of the central VF loss in patients with advanced glaucomatous damage undergoing filtering surgery is generally a rare complication, ${ }^{1,2,4-8}$ although one study in the 1970s reported its prevalence was as high as $14 \% .^{3}$ In a recent prospective study using the HFA 30-2 program in 21 POAG eyes, chronic angle closure glaucoma, or pseudoexfoliation with advanced VF damage (average MD of $-27.9 \pm 2.7 \mathrm{~dB}$ ), no change in the central VF was encountered during the 3-month postoperative follow-up. ${ }^{8}$ The preoperative mean IOP of the subjects $(27 \mathrm{~mm} \mathrm{Hg})$ was higher than that in this study $(19.7 \mathrm{~mm} \mathrm{Hg})$, while reduction rates in IOP after trabeculectomy were comparable (56 vs 53\%,

respectively).

In this study, the effect of trabeculectomy on central VF was prospectively investigated in more detail using the HFA 10-2 program in 27 eyes of 27 OAG patients with advanced VF damage (mean TD of the 10-2 program of -26 to $-27 \mathrm{~dB}$ and $\mathrm{MD}$ of the $30-2$ program of -24 to $-26 \mathrm{~dB}$ ) for 12 months postoperatively. Although this study was not planned to study the frequency of loss of central VA, no case showed either deterioration of VA to $20 / 200^{2,3}$ or loss of central $\mathrm{VF}^{1,6}$ (deterioration of FT to $10 \mathrm{~dB}$ or lower).
There were two eyes with two lines of deterioration in BCVA at 1 month after the surgery compared with the preoperative examination. There was one eye with three lines of deterioration in BCVA (0.5-0.2 in decimal fraction) at 12 months after the surgery compared with that in the preoperative examination. In this eye, IOP was $20 \mathrm{~mm} \mathrm{Hg}$ preoperatively, and $14 \mathrm{~mm} \mathrm{Hg}$ at 12 months after the surgery, and no hypotony and macular splitting were seen. FT, MTD, and $\mathrm{MTD}_{4}$ of this eye got worse than preoperative values at 12 months (21 to $12 \mathrm{~dB},-32$ to $-33 \mathrm{~dB},-26$ to $-33 \mathrm{~dB}$, respectively) without apparent causes. Deterioration in BCVA took place 6 months after surgery in this eye, and the possibility could not be excluded that it reflected surgery-related phenomenon rather than progression of glaucoma.

Several studies have reported that postoperative hypotony was a risk factor for the postoperative central VF loss. ${ }^{2,3,7}$ In this study, six eyes had short-term hypotony and one eye had long-term hypotony, however, none of them had $>2$ lines of deterioration in BCVA (decimal fraction) at 12 months after surgery. Slopes of FT, MTD all, $\mathrm{MTD}_{\text {sup }} \mathrm{MTD}_{\text {inf }}$, $\mathrm{MTD}_{4}, \mathrm{TD}_{29}, \mathrm{TD}_{30}, \mathrm{TD}_{39}$, and $\mathrm{TD}_{40}$ showed no significant correlation with any of the preoperative or postoperative factors.

Macular splitting is speculated to be another risk factor of the 'wipe-out' phenomenon., 2,3,7 If macular splitting is defined as the mean TD value of two adjacent test 
locations of the central four test locations in the upper or lower hemi-fields $\left(\mathrm{TD}_{29}\right.$ and $\mathrm{TD}_{30}$, or $\mathrm{TD}_{39}$ and $\mathrm{TD}_{40}$ ) worse than $-20 \mathrm{~dB}$ and that of the other two locations is better than $-10 \mathrm{~dB}$, then 5 of 27 eyes had macular splitting in the subject of this study, however, none of them had $>2$ lines of deterioration in BCVA (decimal fraction) at 12 months after surgery.

Several studies have reported improvement in VF after trabeculectomy, ${ }^{10,11}$ however, none of the VF parameters of HFA 10-2 program currently studied showed significant improvement while a few of them had positive slope, which were significantly different from 0 $(P<0.05)$ without correction for multiplicity in four eyes (Table 2).

In the subjects of this study, many test points of the 10-2 program showed threshold values of almost $0 \mathrm{~dB}$ in most of the 27 subject eyes. There were only seven test points where TD $>-20 \mathrm{~dB}$ in at least $2 / 3$ (18 of 27) of all subject eyes preoperatively including four test points adjacent to fixation. The point-wise TD value analysis was also performed at those relatively spared points except four test points adjacent to fixation $\left(\mathrm{TD}_{31}, \mathrm{TD}_{41}\right.$, and $\left.\mathrm{TD}_{42}\right)$ and the same results were obtained; that is, there were no significant changes in the VF parameters. It showed similar results when the test points with TD values $>-25 \mathrm{~dB}$ in at least $2 / 3$ of the subject eyes were concerned. The point-wise analysis was designed based on the slope during 12 months of the examination period rather than comparisons between values at the preoperative and each postoperative examination; because the lower TD values deteriorate, the larger its fluctuation become. ${ }^{12}$

To identify pre- or postoperative factors relating to VA and central VF change by trabeculectomy in more detail in advanced stage OAG, larger sample size had been needed. However, it was difficult for a single institute to recruit $>100$ OAG patients with advanced VF damage to whom trabeculectomy was indicated and met the inclusion criteria.

In summary, VA and VF of 27 eyes of 27 OAG patients with advanced central VF damage were prospectively examined for 12 months after trabeculectomy using the HFA 10-2 program. IOP was reduced from approximately $20-10 \mathrm{~mm} \mathrm{Hg}$ over 12 months after trabeculectomy. TD values in the central 10-2 program test locations showed no significant change in that period, but clinically significant decrease in BCVA without apparent causes was found in only $1(4 \%)$ eye at 12 month after the surgery. These results show relative safety of trabeculectomy in OAG eyes with advanced damage regarding sudden loss of central VA or VF and may support validity of surgical intervention to decrease IOP in such eyes if it is indicated.

\section{Summary}

\section{What was known before}

- The prevalence of the wipe-out phenomenon after trabeculectomy is reportedly a rare complication even in the patients with advanced visual field damage. A recent prospective study found no such cases in 3-month follow-up period. However, there have been no prospective studies that assessed central visual field precisely using 10-2 program of Humphrey visual field analyzer in longer postoperative period.

\section{What this study adds}

- The effect of trabeculectomy on central visual field was prospectively investigated using 10-2 program in 27 eyes of 27 open-angle glaucoma patients with advanced visual field damage for 12 months after the surgery. No eyes of the subjects showed either deterioration of visual acuity to $20 / 200$ or less or loss of central visual field. No significant changes found in point-wise investigation in the 10-degree central visual field although significant decrease in visual acuity without apparent causes might occur approximately $5 \%$ of the cases.

\section{Conflict of interest}

The authors declare no conflict of interest.

\section{References}

1 Aggarwal SP, Hendeles S. Risk of sudden visual loss following trabeculectomy in advanced primary open-angle glaucoma. Br J Ophthalmol 1986; 70: 97-99.

2 Costa VP, Smith M, Spaeth GL, Gandham S, Markovitz B. Loss of visual acuity after trabeculectomy. Ophthalmology 1993; 100: 599-612.

3 Kolker AE. Visual prognosis in advanced glaucoma: a comparison of medical and surgical therapy for retention of vision in 101 eyes with advanced glaucoma. Trans Am Ophthalmol Soc 1977; 75: 539-555.

4 Otto J. Loss of point of fixation after glaucoma surgery. Klin Monatsblatter Augenheilkd Augenarztl Fortbild 1957; 131: 178-195.

5 Lichter PR, Ravin JG. Risks of sudden visual loss after glaucoma surgery. Am J Ophthalmol 1974; 78: 1009-1013.

6 Martinez JA, Brown RH, Lynch MG, Caplan MB. Risk of postoperative visual loss in advanced glaucoma. Am J Ophthalmol 1993; 115: 332-337.

7 O'Connell EJ, Karseras AG. Intraocular surgery in advanced glaucoma. Br J Ophthalmol 1976; 60: 124-131.

8 Topouzis F, Tranos P, Koskosas A, Pappas T, Anastasopoulos E, Dimitrakos S et al. Risk of sudden visual loss following filtration surgery in end-stage glaucoma. Am J Ophthalmol 2005; 140: 661-666.

9 Chylack Jr LT, Leske MC, Sperduto R, Khu P, McCarthy D. Lens opacities classification system. Arch Ophthalmol 1988; 106: 330-334

10 Salim S, Paranhos A, Lima M, Shields MB. Influence of surgical reduction of intraocular pressure on regions of the visual field with different levels of sensitivity. Am J Ophthalmol 2001; 132: 496-500.

11 Vuori ML, Vainio-jylhae E, Viitanen TT. Localised changes in glaucomatous visual fields after trabeculectomy. Acta Ophthalmol Scand 2001; 79: 468-471.

12 Heijl A, Lindgren G, Olsson J. Normal variability of static perimetric threshold values across the central visual field. Arc Ophthalmol 1987; 105: 1544-1549. 